\title{
Overlapped Subcarrier Multiplexed WDM PONs Enabled by Burst-Mode Receivers
}

\author{
David V. Plant*, Ziad A. El-Sahn, Jonathan M. Buset, and Bhavin J. Shastri \\ Dept. of Electrical and Computer Engineering, McGill University, Montreal, QC, Canada H3A $2 \mathrm{~A} 7$ \\ *david.plant@mcgill.ca
}

\begin{abstract}
A symmetric WDM PON architecture using an innovative overlapped-SCM scheme that maximizes the spectrum usage of a bandwidth-limited RSOA is demonstrated. In addition, burst mode receivers for this application are discussed.

OCIS codes: (060.2330) Fiber optics communications; (060.4250) Networks
\end{abstract}

\section{Introduction}

Passive optical networks (PONs) are recognized as an economic solution to alleviate the bandwidth bottleneck in access networks by deploying fiber-to-the-home. Reflective semiconductor optical amplifier (RSOA)-based colorless optical network unit (ONU) transmitters have been widely used in wavelength-division multiplexed (WDM) passive optical networks (PONs) [1,2]. Recently, subcarrier multiplexing (SCM) has been proposed for single-feeder (single-wavelength) PONs to mitigate the effect of Rayleigh backscattering [3-5]. In addition to transmission challenges, chip sets for PONs must manage upstream data paths because the network is point-tomultipoint. Using time-division multiple access, multiple ONUs transmit data to the optical line terminal (OLT) in the central office. Due to optical path differences, packets can vary in amplitude and phase to create bursty data. Consequently, this necessitates burst-mode receivers (BMRx) at the OLT. The BMRx front-end is responsible for amplitude recovery, whereas clock and data recovery (CDR) together with fast phase acquisition is performed by a burst-mode CDR (BM-CDR) circuit.

\section{Subcarrier Multiplexed WDM PONs}

Here we introduce an innovative overlapped subcarrier multiplexing (O-SCM) technique that allows a certain overlap between uplink and downlink frequency spectra that permits higher symmetrical bit rates compared to conventional SCM techniques by efficiently utilizing the RSOA bandwidth [6]. The architecture of the O-SCM WDM PON shown in Fig. 1 employs existing infrastructure constraints to reduce potential deployment costs with a single feeder connecting the OLT to an arrayed waveguide grating (AWG)-based remote node (RN). Full duplex communication is achieved over the same feeder at the same wavelength using the proposed O-SCM technique. The downlink is kept at baseband, whereas the uplink is up-converted to an RF subcarrier using the proposed O-SCM. At the ONU side, a fraction of the downlink power is passed to a p-i-n photodiode for detection, and the remaining part seeds a gain saturated RSOA for proper re-modulation to be able to hide the downlink modulation within that of the uplink. For this reason, the extinction ratio (ER) of the uplink is also set higher than that of the downlink [2]. As the RSOA is more saturated, the downlink modulation is highly suppressed allowing a 100\% O-SCM where the subcarrier frequency $f_{s c}$ is equal to the bit rate. Therefore, the splitting ratio of the ONU $1 \times 2$ coupler is carefully selected to maximize the power efficiency of the system (to guarantee that the RSOA is saturated and to have enough power for downlink detection). Selecting the proper ER for both uplink and downlink, and ensuring that the RSOA is deeply saturated, minimizes the crosstalk between the up-converted uplink and the residual downlink (part of the downlink that is not erased by the RSOA), so that the overlap can be tolerated. At the OLT side, the uplink is simply recovered after down-conversion because the residual downlink only acts as an additive high frequency noise that is suppressed by a low pass filter (LPF).

The experimental setup for the O-SCM WDM PON is as follows. At the OLT transmitter, a $1550.08 \mathrm{~nm}$ DFB laser is externally modulated at $1.5 \mathrm{~Gb} / \mathrm{s}$ with a $2^{15}-1$ non-return-to-zero (NRZ) pseudo random binary sequence (PRBS). The downlink ER is set less than $2.5 \mathrm{~dB}$. An erbium-doped fiber amplifier (EDFA) is then used with a variable optical attenuator (VOA) to control the launched power for the bit error rate (BER) measurements. At the ONU side, the RSOA is directly modulated at $1.5 \mathrm{~Gb} / \mathrm{s}$ with a $2^{23}-1 \mathrm{NRZ}$ up-converted $\left(f_{s c}=1.5 \mathrm{GHz}\right) \mathrm{PRBS}$. An RF amplifier and a bias-T are used to drive the RSOA such that the ER is significantly higher than $2.5 \mathrm{~dB}$. A 90/10 coupler is used to tap $10 \%$ of the downlink for reception. The same photodiode (PD), RF amplifier and LPF are used at both ONU and OLT for reception. For the optical distribution network, standard single mode fiber (SMF-28e+) $20.35 \mathrm{~km}$ feeder and $1.5 \mathrm{~km}$ distribution drop fiber, and a $100 \mathrm{GHz}$ AWG are used. Error-free operation is achieved for both downlink and uplink with O-SCM, without dispersion compensation and without forward error correcting (FEC) codes. Based on the power budget analysis and our measurements, we calculate the optimum ONU coupling ratio to minimize the OLT launched power while achieving error-free operation in both directions. The results show that error-free operation (BER $<10^{-10}$ ) in both directions can be achieved with only $-2 \mathrm{dBm}$ of OLT launched power when using a 70/30 ONU coupler. 


\section{ATuA3.pdf}

This O-SCM scheme is therefore best targeted for next generation WDM PONs as it exploits the bandwidth of the RSOA to its maximum compared to conventional SCM techniques. Error-free operation is achieved using realistic launch powers without the need for digital signal processing (DSP) or FEC. Furthermore, the design of the $\mathrm{ONU}$ is inexpensive and simple enough to make it attractive to network operators.

\section{Burst-Mode Receivers}

We have demonstrated novel BM-CDR architectures based on time-oversampling techniques with a hybrid topology of feedback and feed-forward; that is, BM-CDRs built from conventional (SONET) CDRs operated at $2 \times$ the bit rate and clock and phase aligners (CPAs). A block diagram of the BM-CDR is shown in Fig. 2. The idea behind the CPA is based on a simple and effective phase picking algorithm which attempts to detect the packet delimiter (with the byte synchronizers) from the two possibilities of the data samples. The phase picker then selects the right path yielding automatic clock phase acquisition. This design provides low latency and fast response without requiring a reset signal from the network layer.

We have experimentally tested variants of this BM-CDR in various experimental testbeds including: (1) 20-km time-division multiplexing (TDM) gigabit Ethernet PON (GEPON) uplink at $5 \mathrm{~Gb} / \mathrm{s} \mathrm{[7];} \mathrm{(2)} \mathrm{1300-km} \mathrm{deployed} \mathrm{fiber}$ link spanning Montreal to Quebec City and back, at $1.25 \mathrm{~Gb} / \mathrm{s}$ [8]; and (3) 7-user 20-km spectral amplitude coded optical code-division multiple access (SAC-OCDMA) PON uplink at $625 \mathrm{Mb} / \mathrm{s}[9,10]$. In each case, the BM-CDRs have shown to achieve a BER $<10^{-10}$ and a packet loss ratio (PLR) $<10^{-6}$ while featuring instantaneous ( 0 preamble bit) phase acquisition for any phase step ( $\pm 2 \pi \mathrm{rad})$ between successive bursts. In addition, the BMCDRs have BER/PLR sensitivities less than $-24 \mathrm{dBm}$ with negligible burst-mode sensitivity penalty.

Instantaneous phase acquisition can increase the effective throughput of the system by increasing the information rate, and also dramatically improves the physical efficiency of the upstream PON traffic to $99 \%$ for 32 ONUs. The price to pay to obtain instantaneous phase acquisition is faster electronics. On the other hand, our solution is to leverage the design of components for long-haul transport networks using low-complexity, commercial electronics including evaluation kits and field-programmable gate arrays (FPGAs), to provide a cost-effective solution for PON BM-CDRs. These components are typically a generation ahead of the components for multi-access networks. Thus, our solution will scale with the scaling for long-haul networks.

Moving forward, we are developing a more power-efficient BM-CDR architecture based on space-oversampling techniques with multiphase clocks at $10 \mathrm{~Gb} / \mathrm{s}$ [11]. This BM-CDR will achieve instantaneous phase acquisition with no trading-off in the CDR loop-bandwidth. Hence, the BM-CDR could also find applications in future high-speed optical burst/packet switched networks, which may require a cascade of BM-CDRs that each consumes some of the overall jitter budget of the system.

\section{References}

[1] F. Payoux, et al., Optical Fiber Communications (OFC), paper OTuG5, 2007.

[2] F. Payoux, et al., Optical Fiber Communications (OFC), paper OTuC4, 2006.

[3] C. Arellano, at al., Optical Fiber Communications (OFC), paper OTuC1, 2006.

[4] J. M. Fàbrega, et al., European Conference on Optical Communications (ECOC), paper We.1.F.6, 2008.

[5] J.-M. Kang, et al., IEEE Photon. Technol. Lett, 18: 502-504, 2006.

[6] Z. A. El-Sahn, J. M. Buset, and D. V. Plant, Optical Fiber Communications (OFC), paper OMP7, 2011.

[7] B. J. Shastri and D. V. Plant, IEEE J. Sel. Topics Quantum Electron., 16: 1298-1320, 2010.

[8] B. J. Shastri, Y. B. M'Sallem, N. Zicha, L. A. Rusch, S. LaRochelle, and D. V. Plant, J. Opt. Commun. Netw., 2: 1-9, 2010.

[9] Z. A. El-Sahn, B. J. Shastri, M. Zeng, N. Kheder, D. V. Plant, and L. A. Rusch, IEEE/OSA J. Lightw. Technol., 26: 1192-1203, 2008.

[10] B. J. Shastri, Z. A. El-Sahn, M. Zeng, N. Kheder, L. A. Rusch, and D. V. Plant, IEEE Photon. Technol. Lett., 20: 363-365, 2008.

[11] B. J. Shastri, and D. V. Plant, Proc. IEEE Photonics Society Summer Topical Meetings, paper MD4.3, 2010.

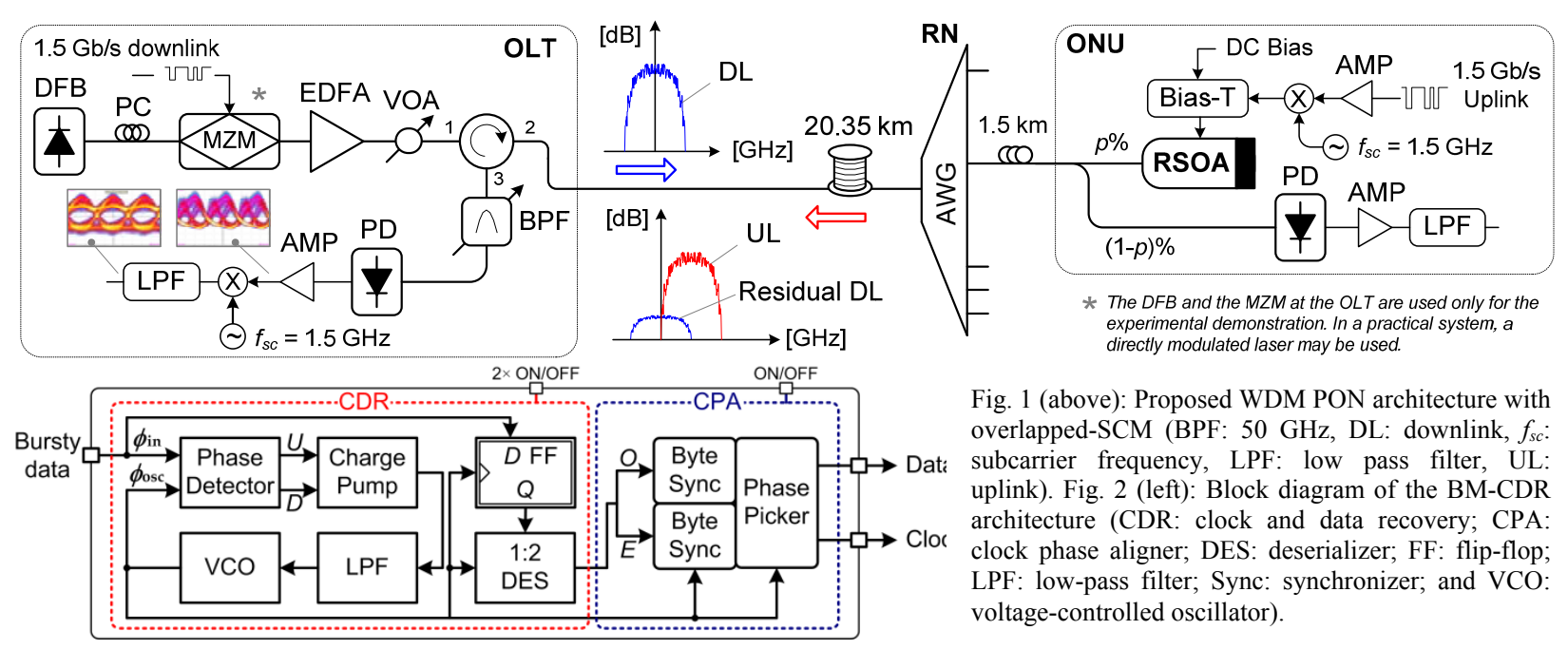

